\title{
Comparing the Use of Echocardiography and Non-Invasive Cardiac System in Measuring the Cardiac Index to Predict Acute Kidney Failure in Patients with Heart Failure
}

\author{
Hari Adityo Nugroho ${ }^{1}$ Antonius Freddy ${ }^{2}$ Cholid Tri Tjahyono ${ }^{3}$ Nanik Setijowati ${ }^{4}$ Ali Haedar ${ }^{2}$ \\ 1.Resident, Emergency Medicine, Faculty of Medicine University of Brawijaya \& Public Hospital dr. Saiful \\ Anwar Malang, Indonesia \\ 2.Teaching Staff and Specialist, Emergency Medicine, Faculty of Medicine University of Brawijaya \& Public \\ Hospital dr. Saiful Anwar Malang, Indonesia \\ 3.Teaching Staff and Specialist, Heart and Blood Vessels, Faculty of Medicine University of Brawijaya \& Public \\ Hospital dr. Saiful Anwar Malang, Indonesia \\ 4.Teaching Staff, Public Health and Statistics, Faculty of Medicine University of Brawijaya \& Public Hospital \\ dr. Saiful Anwar Malang, Indonesia
}

\begin{abstract}
The prevalence of heart failure is $1-2 \%$ of the adult population in developing countries. Decreased kidney function in these patients is influenced by several factors including low Cardiac Output (CO) (Liu, 2008). Cardiac Index (CI) is an important parameter for assessing patients with heart failure since a decrease in $\mathrm{CI}$ in heart failure patients indicates the inadequacy of tissue perfusion, including the kidney, which triggers Acute Kidney Injury (AKI). Thus, it is necessary to study hemodynamic surveillance in patients with heart failure both by using echocardiography which is routinely carried out in the Emergency Room (IGD) of the Saiful Anwar Public Hospital (RSSA) as well as a Non-invasive Cardiac System (NiCAS) monitoring tool to predict AKI in patients
\end{abstract} with heart failure.

Methods: Analytical observations were done in 39 patients with heart failure in the Emergency Room of RSSA to determine the suitability of NiCAS and echocardiography in measuring $\mathrm{CI}$ to predict acute kidney failure in these patients.

Results: The suitability of CI measurements on NiCAS and echocardiography was obtained in 23 samples (59\%) $(\mathrm{p}=0.175)$. Creatinine suitability and Echocardiography CI $\mathrm{R}^{2}$ were 0.0068 . Creatinine suitability and NiCAS CI were tested at $12^{\text {th }}$ hour, since the patient was hospitalized, under linearity conditions resulting in $\mathrm{R}^{2}$ of 0.0024 and $\mathrm{R}^{2} 0.0287$.

Conclusion: Measurement of NiCAS matches measurement with echocardiography in measuring Cardiac Index. However, neither NiCAS nor Echocardiography does not a match in predicting acute kidney failure in patients with acute heart failure.

Keywords: NiCAS, echocardiography, heart failure, kidney failure, Cardiac Output (CO), Cardiac Index (CI)

DOI: $10.7176 / \mathrm{JHMN} / 66-01$

Publication date:September $30^{\text {th }} 2019$

\section{Introduction}

The prevalence of heart failure is estimated to reach $1-2 \%$ of the adult population in developing countries and is mainly experienced by those aged 70 years (Owan, 2006). Heart failure itself can be accompanied by several diseases including renal insufficiency or kidney failure. Decreased filtration rate of the renal glomerulus in heart failure patients is influenced by factors such as low Cardiac Output (CO), increased intra-abdominal pressure, and increased venous pressure (Liu, 2008).

$\mathrm{CO}$ is one of the physiological parameters because it is able to reflect directly and provide a proportional picture of metabolism as a whole. CO indexed by body surface area of Cardiac Index (CI) is an important clinical parameter and is used to assess patients with heart failure and patients with critical illness. Decreased CO and CI in patients with heart failure indicates the inadequacy of tissue perfusion, including perfusion to the kidneys, which triggers acute kidney injury (AKI).

AKI is a condition of sudden kidney function decline within 48 hours. This is marked by an increase in serum creatinine $>0.3 \mathrm{mg} / \mathrm{dl}(>25 \mu \mathrm{mol} / \mathrm{L})$, or a $50 \%$ increase in serum creatinine, or a decrease in urine production (oliguria) $<0.5 \mathrm{ml} / \mathrm{kg}$ /hour for $>6$ hours (AKIN) (Murray, 2007).

A meta-analysis by Smith in 2006 reported that of 80,098 patients treated with heart failure, $63 \%$ experienced worsening kidney function. The deterioration in kidney function is proportional to the increase in mortality. For each increase in serum creatinine level of $0.5 \mathrm{mg} / \mathrm{dL}$, there is an increase in mortality by $15 \%$ (Ronco et al., 2010).

Other studies recommend a scoring system that combines various clinical variables to stratify the risk of patients with acute heart failure. However, this recommendation does not provide specific guidelines for therapy while hemodynamic monitoring can be an immediate indication for intervention. In addition, modalities such as 
echocardiography in CO measurements have several limitations, such as difficulty in obtaining continuous measurements, periodic measurements can be carried out yet personnel who are able to carry out examinations may not always be available, (Huang, 2012)

Taniguchi et al. conducted a study to measure the cardiac output of heart failure patients without using echocardiography, but by using the Non-invasive Cardiac System (NiCAS) as a tool to measure the cardiac output with whole-body impedance cardiography. This tool is able to provide an assessment of cardiac output function as well as cardiac catheterization, but with non-invasive benefits and no complications (Taniguchi, 2013).

The deteriorating kidney function in patients with heart failure can be prevented through good hemodynamic monitoring (cardiac output) and as there have been no studies comparing hemodynamic monitoring devices, the researchers considered it necessary to conduct hemodynamic surveillance studies in patients with heart failure using both echocardiography, which is commonly done in the Emergency Room of RSSA and other non-invasive monitoring tools (NiCAS) to predict the incidence of acute kidney failure in patients with heart failure.

\section{Material and Method}

This study was an observational analytic study to determine the suitability of NiCAS and echocardiography in measuring CI to predict acute kidney failure in patients with heart failure. The inclusion criterion was: patients who came to the emergency room at RSSA aged 40 years old and fulfilled clinical criteria for acute heart failurethe determination of clinical criteria for heart failure was obtained from the anamnesis and physical examination. The exclusion criteria included pregnancy, tumor history, with prognosis $<6$ months, pneumothorax, anaphylactic shock, and acute or critical limb ischemia.

The independent variables in this study were the clinical characteristics of the patient, the results of laboratory measurements, and the CI evaluation of NiCAS and echocardiography. The dependent variable in this study was the diagnosis of heart failure.

The operational definitions in this study were: (1) patients with heart failure, (2) acute renal failure, (3) KDIGO AKI criteria, (4) Cardiac Index, (5) echocardiography, and (6) NiCAS. Patients with heart failure are those who have a history of heart failure (evidenced by medical records or treatment for patients with heart failure). Acute kidney failure is a condition where serum creatinine, urine production, and BUN levels meet the KDIGO AKI criteria. The KDIGO AKI criterion is marked by an increase in creatinine of more than $0.3 \mathrm{mg} / \mathrm{dL}$ from a normal laboratory reference value or urine output of less than $0.5 \mathrm{cc} / \mathrm{kg} /$ hour for a period of 6 hours. Cardiac Index (CI) is Cardiac Output indexed by body surface area. Cardiac Index in this study can be calculated either using echocardiography or NiCAS.

$$
\mathrm{CI}=\frac{\mathrm{CO}}{\mathrm{BSA}}
$$

Echocardiography is the use of ultrasonography to see the function of ejection fraction, measuring CO, CI, and fluid adequacy in patients with heart failure. Ultrasonography used is GE ultrasonography using a sector probe to assess cardiac function. The echocardiography operator was the residents of cardiology. NiCAS is a bioimpedance technology that has been approved by the FDA to measure CI components and is non-invasive. NiCAS was performed when the patient arrived at the emergency room, in the gurney, in the supine position, had no contact with surrounding metal and lower limbs at an angle of $45^{\circ}$ and upper abduction at an angle of $30^{\circ}$ to avoid contact with the skin. Measurements with NiCAS would be done again at T 48 hours after hospitalization from the emergency room and when the patient was discharged from the hospital.

\section{Results}

The results of the analysis showed the comparison of the use of echocardiography and NiCAS in CI measurements to predict acute kidney failure in patients with heart failure according to inclusion criteria. Data collection was carried out within 12 months, from December 2017 to December 2018, after the study was approved and declared ethical. The study took place at the Emergency Department of RSSA. As many as 39 patients met the inclusion criteria. CI measurements were carried out at $12^{\text {th }}$ and $24^{\text {th }}$ hour since the patient was hospitalized using NiCAS and at $12^{\text {th }}$ hour since the patient was hospitalized using echocardiography. Measurements of serum creatinine were carried out at $12^{\text {th }}$ hour and $24^{\text {th }}$ hour since the patient was hospitalized. Informed consent and statement of approval of the research subjects were carried out for all research subjects, namely patients or family members of patients in accordance with the existing ethical principles. The general characteristics of the study subjects included gender, age, and risk factors (Table 1). Numerical data were tested using the normality test, and because the study sample was less than 40, the Sapphiro-Wilk test was used. The Shapiro-Wilk test results showed an abnormal distribution $(\mathrm{p}<0.05)$, so it was continued with the transformation test. After the transformation test was carried out, some of the data still were not normally distributed. The characteristics of the study samples are presented in 
Table 1.

Table 1. Characteristics of Research Subjects

\begin{tabular}{lll}
\hline \multirow{2}{*}{ Sex } & Male & $22(56.4 \%)$ \\
\cline { 2 - 3 } & Female & $17(43.6 \%)$ \\
\hline \multirow{2}{*}{ Age } & Average & 62.28 years old \\
\cline { 2 - 3 } & Minimum-Maximum & $40-86$ years old \\
\hline \multirow{2}{*}{ Hypertension } & Yes & $11(28.2 \%)$ \\
\cline { 2 - 3 } & No & $28(71.8 \%)$ \\
\hline CAD/ACS & Yes & $26(66.7 \%)$ \\
\cline { 2 - 3 } & No & $13(33.3 \%)$ \\
\hline Heart Failure & Yes & $13(33.3 \%)$ \\
NYHA & No & $26(66.7 \%)$ \\
\hline
\end{tabular}

Test on the suitability of Cardiac Index on NiCAS and echocardiography at the $12^{\text {th }}$ hour since the patient was hospitalized was performed using a paired t-test. Corresponding CI measurements in NiCAS and echocardiography were obtained in 23 samples $(59 \%)(\mathrm{p}=0.175)$ as shown in Table 2.

Table 2. The Suitability of Cardiac index between NiCAS and Echocardiography at $12^{\text {th }}$ Hour of Observation

\begin{tabular}{|c|c|c|}
\hline \multirow{2}{*}{ CI NiCAS } & \multicolumn{2}{|c|}{ CI echocardiography } \\
\cline { 2 - 3 } & $<2.5$ or $>4$ & $2.5-4$ \\
\hline$<2.5$ or $>4$ & 12 & 9 \\
\hline $2.5-4$ & 7 & 11 \\
\hline
\end{tabular}

The normality test of creatinine serum and Cardiac Index on echocardiography at the $12^{\text {th }}$ hour showed normal distribution results in which linearity requirements were met with $\mathrm{R}^{2}$ of 0.0068 . This means there was a $0.7 \%$ relationship between $12^{\text {th }}$-hour measurement of creatinine and CI using echocardiography, while $99.3 \%$ was due to other factors. The correlation test showed a non-significant relationship between creatinine levels at the $12^{\text {th }}$ hour and CI echocardiography at the $12^{\text {th }}$ hour since the patient was hospitalized $(\mathrm{p}=0.617, \mathrm{r}=0.083$ ). This is presented in Figure 1.

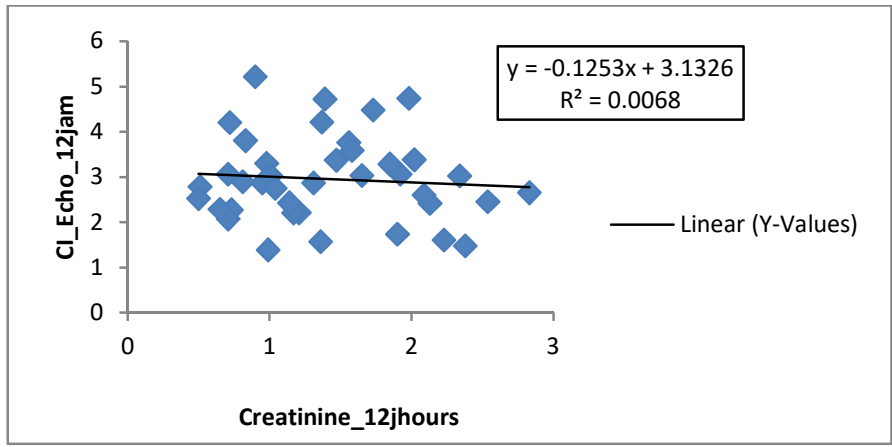

Figure 1. A Correlation Test of Creatinine and Cardiac Index Echocardiography at $12^{\text {th }}$ Hour of Observation

The suitability of creatinine serum and NiCAS Cardiac Index at the $12^{\text {th }}$ hour since the patient was hospitalized was tested with a requirement of linearity of $\mathrm{R}^{2}$ of 0.0024 , which means there was $0.2 \%$ relationship between $12^{\text {th }}$-hour creatinine levels and $12^{\text {th }}$-hour CI, while $99.8 \%$ was due to other factors. The match between creatinine and CI levels was found in 18 subjects $(46 \%)$. The correlation was found not significant between $12^{\text {th }}$ hour creatinine levels and $12^{\text {th }}$-hour CI NiCAS $(\mathrm{p}=0.765, \mathrm{r}=0.049)$. This is presented in Figure 2. 


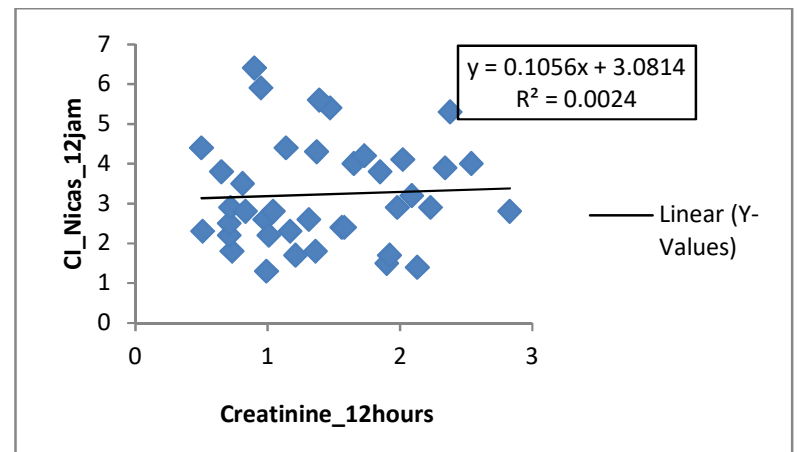

Figure 2. A Correlation Test of Creatinine and Cardiac Index NicAS at $12^{\text {th }}$ Hour of Observation

The suitability of creatinine serum and NiCAS Cardiac Index at the $24^{\text {th }}$ hour since the patient was hospitalized was tested with a requirement of linearity of $\mathrm{R}^{2}$ of 0.0287 , which means there was $2.8 \%$ relationship between $24^{\text {th }}$-hour creatinine levels and $24^{\text {th }}$-hour CI, while $97.2 \%$ was due to other factors. The match between creatinine and CI levels was found in 21 subjects $(54 \%)$ with $\mathrm{p}=1$. The correlation was found significant between $24^{\text {th }}$-hour creatinine levels and $24^{\text {th }}$-hour CI NiCAS $(p=-0.302, r=-0.169)$. This is presented in Figure 3.

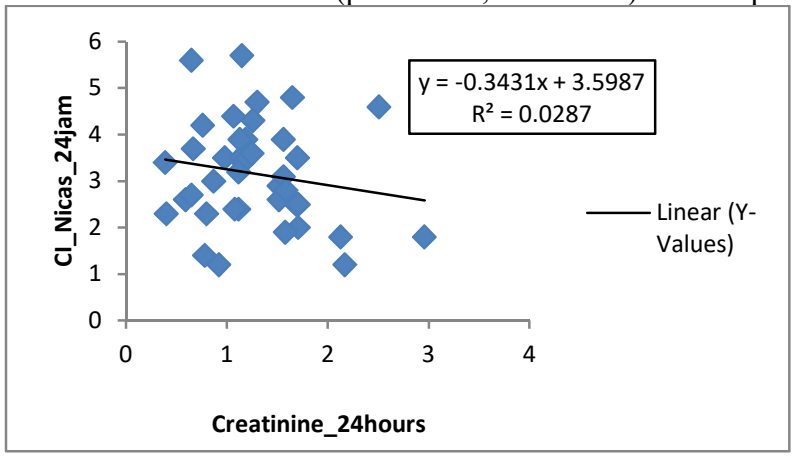

Figure 3. A Correlation Test of Creatinine and Cardiac Index NicAS at $24^{\text {th }}$ Hour of Observation

\section{Discussion}

\section{NiCAS and Echocardiography Cardiac Index}

Echocardiography is now a routine along with a wealth of advance research evidence supporting its use in monitoring CO and CI (Lorne, 2014), while Thoracic Bioimpedance Cardiography (ICG) has been widely used in recent years as an alternative method of non-invasive, sustainable, operator-dependent, and cost-effective in measuring SV and cardiac output. When compared with other cardiac output measurement methods, the most preferred method and the algorithm used by the ICG device have an effect on the correlation coefficient on echocardiography (Fellahi, 2009).

NiCAS is a new ICG device that combines central and peripheral hemodynamic monitoring and aims at measuring non-invasive advanced hemodynamics, which can be useful in the management of anesthesiology and intensive care. NiCAS is also able to capture echocardiogram HR signals to define and measure more accurately the systolic, SV, CO and CI time intervals. ICG has also been proven to be a reliable and repeatable method for monitoring cardiac output in patients with sepsis. However, the routine use of ICG to monitor cardiac output in clinical practice still requires further validation and comparison with other non-invasive hemodynamic tools such as echocardiography (Fellahi, 2009).

This study revealed the suitability of CI measurements between NiCAS and echocardiography in 23 samples $(59 \%)$ and the same result was also found in research by Chen (2014) that there was a correlation between CI measurement results using ICG and echocardiography $(\mathrm{P}<0.05)$; thus, the data measured by ICG can be used as effectively as data from echocardiography in evaluating CI (Chen, 2014).

A report by Leitman (2016) confirmed the same finding that there was a correlation between CI measurement results between NiCAS and echocardiography in patients receiving dobutamine of 0.81 with the CI mean of NiCAS and echocardiography of $2.9 \pm 0.6$ and $2.8 \pm 0.61 / \mathrm{min} / \mathrm{m} 2$, respectively. Siedlecka (2014) confirms that the results of CI measurements on ICG can be used and have similar results in patients with hypertension, heart failure, and rehabilitation after a heart attack.

Variations in CI measurements by ICG correlated well compared with measurement results using echocardiography with $\mathrm{r}=0.88(0.82-0.94), \mathrm{p}<0.001$. ICG can be a reliable, non-invasive method for monitoring 
CI in patients with heart failure (Lorne, 2014).

The disparity of CI measurement data on NiCAS and echocardiography was also found in 16 samples (41\%) of the study. Several factors known to influence ICG readings include scar in the chest area, fluid status that affects vasodilation or vasoconstriction, the use of continuous positive airway pressure that affects cardiac output, and COPD patients due to differences in thoracic cavity volume (Lorne, 2014).

\section{Creatinine Measurement in NiCAS and Echocardiography}

Patients with heart failure often have comorbid kidney disorders and the relationship between the heart and kidneys in this condition has become a quite interesting research topic. Kidney disorder in cases of heart failure, called cardiorenal syndrome, generally occurs due to left ventricular disorders, which results in decreased CO and CI so the kidneys experience hypoperfusion and decreased glomerular filtration rate (Chen, 2017). KDIGO classifies acute kidney disorders (AKI) from stages 1 to 3, based on a decrease in the amount of urine or an increase in serum creatinine levels or both. This criterion has been validated to predict the main mortality rates of patients in hospitals (Kane-Gill, 2015).

This study found no significant correlation between the reduction in CI measured by NiCAS or echocardiography and the increase in serum creatinine measured at $12^{\text {th }}$ and $24^{\text {th }}$ hour since the patient was hospitalized in patients with heart failure. The absence of a significant correlation also made it impossible to find the CI cut-off value in determining the increase in serum creatinine levels in line with some previous studies.

A study by Hanberg (2016) showed a non-significant correlation between decreased CI and renal impairment as indicated by an increase in creatinine in patients with heart failure - the correlation, however, was weak and paradoxical between CI and creatinine increase. The non-linearity test was also not able to find a significant positive correlation between $\mathrm{CI}$ and creatinine. These findings further emphasize that the reduction in $\mathrm{CI}$ is not the main trigger for kidney disorders in heart failure patients (Hanberg, 2016).

Previous studies that also focused on the relationship between CI and kidney function also consistently support the findings in this study. In the majority of cases, no significant correlation was found. Only one study reported a significant relationship between increased CI and worsening renal function (Mullens, 2009).

The glomerular filtration rate is a product of the filtration fraction and renal plasma flow, so it will make sense if all factors that globally worsened perfusion will also worsen the glomerular filtration rate. The concept of decreased CI, which causes kidney disorders in the form of decreased eGFR, increases creatinine and urea seems to be too simplified because the kidneys cannot directly feel or respond to the amount of blood pumped into the ascending aortic by the heart (cardiac output). Autoregulation in the kidneys, both through the tubuloglomerular feedback pathway and myogenic autoregulator, is able to maintain renal perfusion and the filtration rate constantly under reduced CI conditions (Hanberg, 2016).

The study by Nohria (2009) also explained that the reduction in CI was not a major trigger for decreased kidney function (defined as an increase in serum creatinine $>0.3 \mathrm{mg} / \mathrm{dL}$ ) and only had a weak correlation $(\mathrm{p}<$ 0.01). The main factors triggering kidney dysfunction in patients with heart failure is (Nohria, 2009):

1. Patients as the study subjects tended to be elderly ( $>65$ years) and had a higher prevalence of comorbidities, such as hypertension and diabetes, which caused intrinsic renal disorders and heart failure.

2. The presence of hypertension and diabetes, especially hypertension, interferes with autoregulation of renal blood flow, made this organ more vulnerable to deteriorating function during heart failure therapy. In patients with heart failure, the renal autoregulation curve moves to the right, which causes a decrease in Mean Arterial Pressure (MAP) as a consequence of decreased blood flow to the kidneys, vasodilation drugs, or diuretics that decrease intraglomerular pressure and glomerular filtration rate, which can affect kidney function in normal subjects.

3. Hypertension and diuretic drugs, especially thiazide, combined with diuretic loops are strongly correlated with worsening renal function.

\section{Limitation}

This study has several limitations, namely:

1. Impaired kidney function is reflected by serum creatinine measurements at certain times $\left(12^{\text {th }}\right.$ and $24^{\text {th }}$ hour since the patient was hospitalized); most patients with heart failure are assumed to have different kinetic variations in the creatinine base values.

2. Glomerular filtration rate (where creatinine is a measurement element in it) in patients with heart failure is affected by a variety of risk factors such as hypertension and diabetes mellitus that can damage the kidneys intrinsically before getting involved in this study and this may have dissimulated the correlation between "true" heart failure and kidney dysfunction and CI.

3. The creatinine parameters used in this study were not dominantly influenced by kidney damage such as damage to tubular function, but rather to muscle damage; thus, future studies need to examine the relationship between the decrease in CI with kidney function parameters such as tubular damage (like cystatin C). 


\section{Conclusions}

- The measurement of NiCAS as a non-invasive impedance method was suitable in measuring cardiac index as it showed the same results as shown by echocardiography.

- Echocardiography was suitable to be used in cardiac index measurements to predict acute kidney failure in patients with acute kidney failure in 20 samples $(\mathrm{p}=0.648)$. Statistical tests showed that there was no significant relationship between CI echocardiography and increased serum creatinine levels.

- NiCAS was suitable to be used in cardiac index measurements at the $12^{\text {th }}$ hour and the $24^{\text {th }}$ hour since the patient was hospitalized. It was showing the suitability of 18 samples at the $12^{\text {th }}$ hour since the patient was hospitalized. However, at the $24^{\text {th }}$ hour since the patient was hospitalized, the test showed no significant relationship.

- There was no matching cardiac index measurement using echocardiography or using NiCAS in predicting acute kidney failure in patients with acute heart failure.

\section{Suggestions}

- The Emergency Room of Syaiful Anwar Public Hospital (RSSA) can consider the use of NiCAS as a noninvasive monitoring tool for cardiac function because it has the same reliability as echocardiography.

- Further studies on the relationship between a decrease in cardiac index and the main parameters of kidney function such as tubular damage (such as cystatin C) must be done.

\section{References}

Abuelo, J.G., 2007. Normotensive ischemic acute renal failure. New England Journal of Medicine, 357(8), pp.797805.

Berton, C. and Cholley, B., 2002. Equipment review: new techniques for cardiac output measurement-oesophageal Doppler, Fick principle using carbon dioxide, and pulse contour analysis. Critical Care, 6(3), p.216.

Bougault, V., Lonsdorfer-Wolf, E., Charloux, A., Richard, R., Geny, B. and Oswald-Mammosser, M., 2005. Does thoracic bioimpedance accurately determine cardiac output in COPD patients during maximal or intermittent exercise?. Chest, 127(4), pp.1122-1131.

Bui, A.L., Horwich, T.B. and Fonarow, G.C., 2011. Epidemiology and risk profile of heart failure. Nature Reviews Cardiology, 8(1), p.30.

Chittineni, H., Miyawaki, N., Gulipelli, S. and Fishbane, S., 2007. The risk for acute renal failure in patients hospitalized for decompensated congestive heart failure. American journal of nephrology, 27(1), pp.55-62.

Forman, D.E., Butler, J., Wang, Y., Abraham, W.T., O'Connor, C.M., Gottlieb, S.S., Loh, E., Massie, B.M., Rich, M.W., Stevenson, L.W. and Young, J.B., 2004. Incidence, predictors at admission, and impact of worsening renal function among patients hospitalized with heart failure. Journal of the American College of Cardiology, 43(1), pp.61-67.

Hett, D.A. and Jonas, M.M., 2003. Non-invasive cardiac output monitoring. Current Anaesthesia \& Critical Care, 14(4), pp.187-191.

Isselbacher, E.M., Pasternak, R.C., Criqui, M.H., Benjamin, E.J., Fowkes, F.G.R., McCullough, P.A., Wolf, P.A. and Zheng, Z.J., 2012. Atherosclerotic vascular disease conference: writing group I: epidemiology. Circulation, 109(21), pp.2605-2612.

Kaufmann, P.A., Jiang, Z., Attenhofer, C., Linka, A. and Mandinov, L., 2000. In vitro validation of volumetric blood flow measurement using Doppler flow wire. Ultrasound in medicine \& biology, 26(8), pp.1301-1310.

Kemp, C.D. and Conte, J.V., 2012. The pathophysiology of heart failure. Cardiovascular Pathology, 21(5), pp.365-371.

Liu, P.P., 2008. Cardiorenal syndrome in heart failure: a cardiologist's perspective. Canadian Journal of Cardiology, 24, pp.25B-29B.

Lavdaniti, M., 2008. Invasive and non-invasive methods for cardiac output measurement. International Journal of Caring Sciences, 1(3), p.112.

Matthew, B., Singh, P.M., Dhillon, M.S. and Hunt, K.R., Tosense Inc, 2016. Body-worn sensor for characterizing patients with heart failure. U.S. Patent 9,259,183.

Murray, P.T., Roy, A.K., Mc Gorrian, C., Treacy, C., Kavanaugh, E., Brennan, A., and Mahon, N.G., 2013. A comparison of traditional and novel definitions (RIFLE, AKIN, and KDIGO) of acute kidney injury for the prediction of outcomes in acute decompensated heart failure. Cardiorenal medicine, 3(1), pp.26-37.

Nowak, R.M., Nanayakkara, P., DiSomma, S., Levy, P., Schrijver, E., Huyghe, R., Autunno, A., Sherwin, R.L., Divine, G. and Moyer, M., 2014. Noninvasive hemodynamic monitoring in emergency patients with suspected heart failure, sepsis and stroke: the PREMIUM registry. Western Journal of Emergency Medicine, 15(7), p.786.

Owan, T.E., Hodge, D.O., Herges, R.M., Jacobsen, S.J., Roger, V.L. and Redfield, M.M., 2006. Secular trends in renal dysfunction and outcomes in hospitalized heart failure patients. Journal of cardiac failure, 12(4), 
pp.257-262.

Packer, M., Abraham, W.T., Mehra, M.R., Yancy, C.W., Lawless, C.E., Mitchell, J.E., Smart, F.W., Bijou, R., O'Connor, C.M., Massie, B.M. and Pina, I.L., 2006. Utility of impedance cardiography for the identification of short-term risk of clinical decompensation in stable patients with chronic heart failure. Journal of the American College of Cardiology, 47(11), pp.2245-2252.

Philipp K., Hafer, C., Lukasz, A., Lichtinghagen, R., Brand, K., Fliser, D., Faulhaber-Walter, R. and Kielstein, J.T., 2010. Serum neutrophil gelatinase-associated lipocalin at inception of renal replacement therapy predicts survival in critically ill patients with acute kidney injury. Critical care, 14(1), p.R9.

Prabhu, M., 2007. Cardiac output measurement. Anaesthesia \& Intensive Care Medicine, 8(2), pp.63-66.

Prentice, D. and Sona, C., 2006. Esophageal Doppler monitoring for hemodynamic assessment. Critical care nursing clinics of North America, 18(2), pp.189-93.

Roger, V.L., 2013. Epidemiology of heart failure. Circulation research, 113(6), pp.646-659.

Ronco, C., Goh, C.Y., Haase-Fielitz, A., Cruz, D.N.. and Haase, M., 2010. Early biomarkers of renal injury. Congestive heart failure, 16, pp.S25-S31.

Sachin, S.S., Sabool, S.S., Raman, A. and Chakravartis, R.M., 2010. New Biomarkers in Acute Kidney Injury. Nephrology and Clinical Chemistry: The Essential Link, p.49.

Sean, B.M., George, C. and Bellomo, R., 2008. A comparison of the RIFLE and AKIN criteria for acute kidney injury in critically ill patients. Nephrology Dialysis Transplantation, 23(5), pp.1569-1574.

Smith, G.L., Lichtman, J.H., Bracken, M.B., Shlipak, M.G., Phillips, C.O., DiCapua, P. and Krumholz, H.M., 2006. Renal impairment and outcomes in heart failure: systematic review and meta-analysis. Journal of the American College of Cardiology, 47(10), pp.1987-1996.

Springfield, G.S., and Wang, D.J., 2006. Impedance cardiography: more questions than answers. Current heart failure reports, 3(3), pp.107-113.

Taniguchi, Y., Emoto, N., Miyagawa, K., Nakayama, K., Kinutani, H., Tanaka, H., Shinke, T. and Hirata, K.I., 2013. Noninvasive and simple assessment of cardiac output and pulmonary vascular resistance with wholebody impedance cardiography is useful for monitoring patients with pulmonary hypertension. Circulation Journal, 77(9), pp.2383-2389.

Tanino, Y., Shite, J., Paredes, O.L., Shinke, T., Ogasawara, D., Sawada, T., Kawamori, H., Miyoshi, N., Kato, H., Yoshino, N. and Hirata, K.I., 2009. Whole body bioimpedance monitoring for outpatient chronic heart failure follow up. Circulation Journal, 73(6), pp.1074-1079.

Yancy, C.W., Jessup, M., Bozkurt, B., Butler, J., Casey, D.E., Drazner, M.H., Fonarow, G.C., Geraci, S.A., Horwich, T., Januzzi, J.L. and Johnson, M.R., 2013. 2013 ACCF/AHA guideline for the management of heart failure: a report of the American College of Cardiology Foundation/American Heart Association Task Force on Practice Guidelines. Journal of the American College of Cardiology, 62(16), pp.e147-e239 